\title{
Métodos de auxílio multicritério à decisão aplicados a problemas de PCP: Mapeamento da produção em periódicos publicados no Brasil
}

\author{
Multicriteria decision aid methods applied to PPC problems: \\ A mapping of papers published in Brazilian journals
}

\author{
Dey Salvador Sanchez Rodriguez ${ }^{1,2}$ \\ Helder Gomes Costa ${ }^{2}$ \\ Luiz Felipe Roris Rodriguez Scavarda do Carmo'
}

\begin{abstract}
Resumo: O presente trabalho tem por objetivo estabelecer um mapeamento da produção científica divulgada em importantes periódicos publicados no Brasil sobre a aplicação de Métodos de Auxílio Multicritério à Decisão (AMD) à modelagem de problemas de Planejamento e Controle da Produção. O mapeamento contemplou 32 artigos, que foram selecionados criteriosamente, tendo como base a lista de periódicos científicos oferecida no Scielo e em importantes sociedades científicas brasileiras no âmbito da Engenharia de Produção. Os resultados permitiram indicar, entre outros aspectos, os periódicos que mais publicam neste contexto, os métodos de AMD mais usados nesta interface, os setores de produção nos quais as aplicações ocorreram e os tópicos de PCP mais abordados. Também foram destacados os aspectos de PCP que ainda carecem de estudos que envolvam métodos multicritério. Palavras-chave: AMD. PCP. ELECTRE. AHP. Revisão bibliográfica.
\end{abstract}

\begin{abstract}
The objective of this study is to map the Brazilian scientific production based on Brazilian journal publications on the implementation of Multicriteria Decision Aid (MCDA) to model Production Planning and Control problems. It consisted of 32 papers that were systematically selected from journals indexed in the Scielo database and also from a list of journals suggested by major Brazilian Industrial Engineering research societies. The results revealed, among other aspects, the journals that publish the most on this topic, the most often used MCDA methods in this interface, the production sectors in which the applications took place, and the most commonly discussed PPC topics. This paper also highlights the PPC aspects that need further research including MCDA methods.
\end{abstract}

Keywords: MCDA. PPC. ELECTRE. AHP. Literature review.

\section{Introdução}

Os sistemas de Planejamento e Controle da Produção (PCP) desempenham um importante papel na busca contínua da melhoria no uso dos recursos de produção. Paralelamente, a modelagem de problemas de decisão, sob a ótica de múltiplos critérios, tem tido uma profusão no âmbito da engenharia de produção. Neste âmbito, Roy (1990) destaca a consolidação de duas vertentes do conhecimento: a tomada de decisão multicritério, em inglês Multicriteria Decision Making (MCDM); e o auxílio multicritério à decisão (AMD), em inglês Multicriteria Decision Aid (MCDA). A primeira vertente (MCDM) dedica-se à modelagem de problemas de decisão baseada no emprego de modelos matemáticos à otimização, os quais requerem que o modelo seja robusto o suficiente para suportar validações axiomáticas, o que é um fator limitante para a sua aplicação à modelagem em certas situações envoltas em subjetividade, ambiguidades e incertezas. Já a segunda vertente (MCDA) se apresenta como uma alternativa para a modelagem de problemas em que subjetividade, incertezas e ambiguidades estejam presentes, pois, ao abrir mão da necessidade de validações axiomáticas presentes em modelos de otimização, tem-se a possibilidade de incorporar tais elementos ao modelo, aproximando-o mais da realidade. Roy (1990) destaca ainda que, se por um lado os Métodos de MCDA abrem mão de validações axiomáticas presentes no MCDM, estes métodos se aprofundam na validação no âmbito de testes associados à análise de coerência, identificando

\footnotetext{
${ }^{1}$ Departamento de Engenharia Industrial - DEI, Pontifícia Universidade Católica do Rio de Janeiro - PUC-Rio, Rua Marquês de São Vicente, 225, Gávea, CEP 22453-900, Rio de Janeiro, RJ, Brasil, e-mail: deysalvador@vm.uff.br; lf.scavarda@puc-rio.br

2 Departamento de Engenharia de Produção, Centro Tecnológico, Universidade Federal Fluminense - UFF, Rua Passo da Pátria, 256, Bloco D, São Domingos, CEP 24210-240, Niterói, RJ, Brasil, email: hgcc@latec.uff.br Recebido em 5/3/2011 — Aceito em 21/9/2012
} 
e tratando ambiguidades e conflitos no processo decisório.

A presença de publicações nos contextos do MCDM e do MCDA pode ser observada no âmbito da literatura científica associada ao PCP. No entanto, os relatos destas modelagens estão geralmente distribuídos em diferentes ambientes de difusão científica. Este fato dificulta a construção de uma visão integrada e abrangente a respeito do desenvolvimento desta integração, assim como pode induzir uma perda de visão global sobre o assunto. O presente artigo está delimitado nas aplicações de MCDA, definido conforme Roy (1990), ao PCP. Apesar da existência de um amplo número de trabalhos dedicados a este escopo, a literatura acadêmica carece de estudos que revisem sistematicamente o que se tem feito de pesquisa nesta interface, sendo a importância de se levantar o estado da arte em MCDA destacada em Ehrgott, Figueira e Greco (2010).

Neste contexto, o presente artigo tem por objetivo oferecer um mapeamento da produção científica publicada em periódicos nacionais, relacionada ao uso de métodos de auxílio multicritério à decisão, aplicados a problemas de PCP. Com esta pesquisa, espera-se, por meio de uma revisão sistemática da literatura, contribuir para a consolidação do conhecimento sobre as áreas de atuação ou aplicabilidade destes métodos dentro do contexto do PCP no Brasil. Vale destacar que levantamentos do estado da arte em MCDA são oferecidos em Hwang e Yoon (1981), Figueira, Greco e Ehrgott (2005) e Ehrgott, Figueira e Greco (2010), porém não focam uma revisão sistemática da literatura acadêmica, nem a interface da aplicação de seus métodos no PCP, proposta do presente artigo. Já Behzadian et al. (2010) e Subramanian e Ramanathan (2012) realizam revisões sistemáticas da literatura, porém focam métodos específicos (Promethee e AHP, respectivamente) e usam, como base, apenas um conjunto específico de periódicos internacionais.

\section{Auxílio multicritério à decisão (mcda)}

Figueira, Greco e Ehrgott (2005) e Polatidis et al. (2006) consideram que os métodos de MCDA mais tradicionais e estabelecidos podem ser classificados em Métodos de Superação (também denotados em português por Métodos de Sobreposição), em inglês, outranking, e em francês, surclassement, e em Teoria da Utilidade Multiatributo (em inglês, Multiattribute Utilityand Value Theory). No âmbito dos Métodos de Superação, um conjunto finito de alternativas $(A)$ é valorado à luz de um conjunto de critérios $(F)$, construindo-se relações de sobre classificação não compensatórias entre as alternativas. Estas relações caracterizam-se pela não criação de uma função única que agregue os desempenhos alcançados, considerando todos os critérios. Já os métodos no âmbito da Teoria da Utilidade Multiatributo caracterizam-se por buscar uma função que agregue diferentes funções de utilidade em uma função única (COSTA et al., 2007).

A Tabela 1 apresenta, em sua primeira coluna, os principais métodos de auxílio multicritério à decisão, encontrados na literatura acadêmica com base em

Tabela 1. Principais métodos de MCDA.

\begin{tabular}{|c|c|c|}
\hline Método & Classificação & Referências seminais \\
\hline Electre & Método de superação & $\begin{array}{l}\text { ELECTRE I (ROY, 1968); ELECTRE II } \\
\text { (ROY; BERTIER, 1971); ELECTRE III } \\
\text { (ROY; HUGONNARD, 1981); ELECTRE IV } \\
\text { (ROY; HUGONNARD, 1981); ELECTRE IS } \\
\text { (ROY; SKALKA, 1985); ELECTRE TRI (YU, 1992; } \\
\text { MOUSSEAU; SLOWINSKI; ZIELNIEWICZ, 2000) }\end{array}$ \\
\hline Promethee & Método de superação & $\begin{array}{l}\text { Brans, Mareschal e Vincke (1984) e Brans, Vincke e } \\
\text { Mareschal (1986) }\end{array}$ \\
\hline Regime & Método de superação & Hinloopen, Nijkamp e Rietveld (1983) \\
\hline $\begin{array}{l}\text { Multiattribute Utility } \\
\text { Theory (MAUT) }\end{array}$ & $\begin{array}{l}\text { Teoria da Utilidade } \\
\text { Multiatributo }\end{array}$ & Fishburn (1970) e Keeney e Raiffa (1976) \\
\hline $\begin{array}{l}\text { Simple Multi Attribute Rating } \\
\text { Technique (SMART) }\end{array}$ & $\begin{array}{l}\text { Teoria da Utilidade } \\
\text { Multiatributo }\end{array}$ & Edwards (1977) \\
\hline Analytic Hierarchy Process (AHP) & $\begin{array}{l}\text { Teoria da Utilidade } \\
\text { Multiatributo }\end{array}$ & Saaty (1977) e Saaty (1980) \\
\hline Analytic Network Processes (ANP) & $\begin{array}{l}\text { Teoria da Utilidade } \\
\text { Multiatributo }\end{array}$ & Saaty (1996) \\
\hline $\begin{array}{c}\text { Measuring Attractiveness by a } \\
\text { Categorical Based Evaluation } \\
\text { Technique (MACBETH) }\end{array}$ & $\begin{array}{l}\text { Teoria da Utilidade } \\
\text { Multiatributo }\end{array}$ & Bana e Costa e Vansnick (1994) \\
\hline
\end{tabular}


Figueira, Greco e Ehrgott (2005) e Polatidis et al. (2006). A segunda coluna desta tabela oferece a classificação de cada um destes métodos com base nestes mesmos autores, enquanto que a terceira coluna apresenta as referências seminais de cada um destes métodos.

Figueira, Greco e Ehrgott (2005) citam ainda o Tomaso (MARICHALA; MEYERB; ROUBENS, 2006) e Verbal Decision Analysis, - VDA (LARICHEV; MOSHKOVICH, 1997), porém destacam que ambos adotam abordagens não tradicionais e menos utilizadas, o que também ocorre com Zapros (LARICHEV; MOSHKOVICH, 1995) e VIP Analysis (CLÍMACO et al., 2009). Há ainda alguns métodos criados por pesquisadores brasileiros, tais como o Thor, apresentado em Gomes (2005), e o Todim (Tomada de Decisão Interativa e Multicritério), reportado em Gomes e Lima (1992).

Os métodos de MCDA têm sido aplicados em diversos tipos de problemas, como nas áreas de finanças, agronegócios, ecologia, saneamento básico, planejamento civil e militar, segurança e política pública, educação, medicina, biologia, planejamento energético, telecomunicações, desenvolvimento sustentável e planejamento e controle da produção; sendo este último o foco deste artigo.

\section{Abordagem utilizada}

Para que o objetivo deste artigo fosse atingido, efetuou-se um levantamento buscando artigos em periódicos publicados no Brasil, para selecionar trabalhos que reportassem a utilização de métodos multicritério no âmbito do PCP. A escolha deste recorte (artigos publicados em periódicos no Brasil) se deve à importância do PCP na fase atual de desenvolvimento econômico no Brasil, que induz a necessidade de adoção de técnicas de gestão mais elaboradas.

Rowley e Slack (2004) afirmam que uma importante ferramenta para a seleção de artigos de periódicos científicos é o uso da base de dados on-line. Inicialmente, foram consultados os periódicos cadastrados no Scielo Brasil, que contemplava, à época da pesquisa, periódicos da área de Engenharia de Produção cadastrados a partir de 1999. Este fato, associado ao propósito de se mapear os textos atualizados, levou à decisão de se limitar a pesquisa à produção científica dos últimos 13 anos (1999-2011). Além das coleções de periódicos cadastradas no Scielo, foram também consultados periódicos citados nos portais da Associação Brasileira de Engenharia de Produção (ABEPRO) e da Sociedade Brasileira de Pesquisa Operacional (SOBRAPO), dada a representatividade no Brasil da ABEPRO, no âmbito do termo Planejamento e Controle da Produção, e da SOBRAPO, no contexto do Auxílio Multicritério à Decisão. A revisão foi limitada quanto ao uso de fontes de informação, utilizando somente periódicos científicos, excluindo assim informações oriundas da chamada "grayliterature" (ROTHSTEIN; HOPEWELL, 2009), como de jornais profissionais (industry magazines), revistas de indústrias, livros texto, workingpapers e conferências, o que é um procedimento normalmente utilizado na literatura em trabalhos que objetivam realizar revisões sistemáticas da literatura sobre um tema específico (e.g. BEHZADIAN et al., 2010; SUBRAMANIAN; RAMANATHAN, 2012). A concentração em periódicos científicos se justifica pelo fato de que eles são geralmente utilizados por acadêmicos e profissionais para adquirir conhecimento e disseminar novos resultados, representando o maior nível de investigação (NORD; NORD, 1995; NGAI; WAT, 2002; VAN RAAN, 2003; NGAI; XIU; CHAU, 2009; FETSCHERIN; VOSS; GUGLER, 2010; TAVARES NETO; GODINHO FILHO, 2012).

Para a seleção dos artigos, executou-se uma pesquisa avançada, utilizando expressões booleanas ("AND" e "OR"), que permitem a combinação de palavras-chave no sentido de aproximar-se o melhor possível de um termo específico, o que é preconizado em Rowley e Slack (2004). O processo de busca foi baseado na utilização das expressões mais comuns associadas ao termo "auxílio multicritério à decisão" e também com base nos principais métodos existentes, estes destacados na seção 2 deste artigo. Assim foi usada a seguinte expressão: ((multicritério OR multicriteria OR "auxílio multicritério à decisão" OR "multicriteria decision aid" OR AMD OR MCDA OR ELECTRE OR PROMOTHE OR REGIME OR MAUT OR SMART OR AHP OR ANP OR TOMASO OR VDA OR ZAPROS OR VIP MACBETH OR THOR OR TODIM) AND ("Production Planing and Control" OR "Planejamento e Controle da Produção" OR PCP OR PPC).

Com esta abordagem de inclusão de artigos, 58 artigos foram selecionados para análise por meio de sua leitura completa pelos pesquisadores envolvidos. Esta análise excluiu os artigos que não tratam do tema com relevância e criticidade, ou seja, que apresentam apenas comentários superficiais sobre o assunto, não estando diretamente relacionados ao escopo da pesquisa em questão, ou, então, não tratam de uma abordagem na qual se aplicava algum método de MCDA à modelagem de algum problema de PCP. Como resultado deste critério de exclusão, apenas 32 artigos foram selecionados para a revisão sistemática proposta, cujos resultados são apresentados na próxima seção.

\section{Resultados da pesquisa}

A análise dos 32 artigos selecionados é apresentada nas próximas subseções. Apresenta-se inicialmente uma breve descrição do conteúdo de cada um destes artigos, organizada por áreas do PCP; e, na 
sequência, estatísticas referentes a eles e uma síntese dos resultados obtidos.

\subsection{Análises das publicações por área de aplicação}

Primeiramente, com base na análise de livros textos sobre PCP publicados no Brasil (RUSSOMANO, 1996; CORREA; GIANESE; CAON, 2007; LUSTOZA et al., 2008; TUBINO, 2009; FERNANDES; GODINHO FILHO, 2010), construiu-se a Tabela 2. Esta tabela indica os conteúdos tratados nestes livros textos.

A análise comparativa destes textos, associada à análise de livros textos internacionais (BUFFA, 1976; ELSAYED; BOUCHER, 1994; SIPPER; BULFIN, 1997; VOLLMANN et al., 2004; CHAPMAN, 2006) e da tabela das áreas de classificação de artigos do periódico Production Planning and Control, publicado pela editora Francis \& Taylor, conduziu à seguinte classificação de temas de PCP, a qual foi utilizada na pesquisa: Controle da Produção; Gestão da cadeia de suprimentos; Gestão da Manutenção; Gestão de Projetos; Job Shop; Localização; Logística de distribuição; Modelos de estoques; Planejamento agregado; Planejamento da capacidade; Planejamento das necessidades de materiais; Planejamento de Layout (arranjos físicos); Previsão de demanda; Programa Mestre de Produção (MPS); Programação da Produção.
$\mathrm{Na}$ análise de cada um dos artigos, foram elaboradas resenhas que consideraram principalmente três aspectos: a problemática específica no contexto do PCP; a metodologia multicritério usada no desenvolvimento do trabalho; e o contexto da aplicação da metodologia. A seguir, apresentam-se estas agrupadas de acordo com as áreas de PCP definidas no parágrafo anterior.

\subsubsection{Controle da produção}

A produção científica analisada reporta um conjunto de nove aplicações de MCDA efetuadas no âmbito do controle da produção. Destes artigos, seis baseiam-se no método AHP, indicando a concentração do uso deste método nesta categoria de problema. SMART e MAUT também são empregados em controle da produção.

Sellitto e Mendes (2006) reportam a aplicação do AHP à avaliação de desempenho de uma fábrica de equipamentos eletrônicos com o propósito de determinar a importância relativa de cada critério e assim definir as prioridades de produção.

Salomon e Shimizu (2006) utilizam o AHP para modelar o controle da produção. O modelo proposto considerou quatro alternativas de ação e trabalhou com três critérios: probabilidade de distúrbio; variabilidade do processamento; e frequência de reprogramação.

Aguiar e Salomon (2007) abordam a integração do AHP aos Conjuntos Fuzzy com o objetivo de

Tabela 2. Temas abordados em livros de PCP publicados no Brasil.

\begin{tabular}{|c|c|}
\hline Livro texto & Conteúdos abordados \\
\hline Lustoza et al. (2008) & $\begin{array}{l}\text { Previsão de demanda; Gestão de estoques; Planejamento agregado; Plano mestre da } \\
\text { produção; Planejamento das necessidades dos materiais (MRP); Programação detalhada } \\
\text { da produção; Sistemas de controle da produção; Logística; Sistemas de informação; } \\
\text { Planejamento da capacidade; Planejamento e controle da produção em serviços. }\end{array}$ \\
\hline $\begin{array}{c}\text { Fernandes e } \\
\text { Godinho Filho (2010) }\end{array}$ & $\begin{array}{l}\text { Previsão de demanda; Planejamento agregado e Planejamento da capacidade no médio } \\
\text { prazo; Planejamento desagregado e Controle de itens de leadtime longo; Programa mestre } \\
\text { de produção (MPS) e Análise de capacidade no nível do MPS; Sistemas de coordenação } \\
\text { de ordens; Detalhamento de alguns sistemas de coordenação de ordens e Análise de } \\
\text { capacidade no curto prazo; Controle de estoques; Controle de chão de fábrica (SFC) } \\
\text { destacando a programação de operações (Scheduling); Estratégias de planejamento e } \\
\text { controle da produção; Tecnologia de grupo e manufatura celular; Coordenação de projetos } \\
\text { com PERT/CPM; Balanceamento de linha de montagem. }\end{array}$ \\
\hline $\begin{array}{l}\text { Correa, Gianese e } \\
\text { Caon (2007) }\end{array}$ & $\begin{array}{l}\text { Sistemas de administração da produção; Conceitos de gestão de } \\
\text { estoques; MRP - planejamento de necessidades materiais; sistema MRP } \\
\text { ii - manufacturingresourcesplanning; S\&OP - planejamento de vendas e operações (sales } \\
\text { and operations planning); MPS - planejamento-mestre da produção; Gestão de demanda; } \\
\text { Planejamento de capacidade; Shop floor control (SFC); Manufacturing execution systems } \\
\text { (MES); Sistema de programação da produção com capacidade finita; Sistemas híbridos com } \\
\text { o MRP II/ERP; Sistemas ERP - Enterprise Resource Planning; Implantação do sistema. }\end{array}$ \\
\hline Tubino (2009) & $\begin{array}{l}\text { Previsão da demanda; Planejamento estratégico da produção; Planejamento mestre da } \\
\text { produção; Programação da produção; Modelos de controle de estoques; Sequenciamento } \\
\text { da programação da produção; Programação puxada da produção - Sistema Kanban; } \\
\text { Emissão, liberação, acompanhamento. }\end{array}$ \\
\hline
\end{tabular}


implementar as práticas de utilização de FMEA (Failure Modeand Effect Analysis). O AHP é aplicado para a priorização das irregularidades quanto à gravidade de sua ocorrência, ao passo que os Conjuntos Fuzzy são aplicados para a avaliação do desempenho da utilização de FMEA. O estudo trabalhou com informações obtidas junto a uma empresa fornecedora de primeiro nível na cadeia automotiva e localizada no interior do Estado de São Paulo (Brasil).

Rafaeli e Müller (2007) constroem um conjunto de indicadores de gestão a partir dos resultados apresentados pela aplicação de metodologias de gestão já praticadas pela organização, sendo elas: EVA (valor econômico agregado); TOC (teoria das restrições); TQM (qualidade total); BSC (balanced score card); e CI (capital intelectual). Estes indicadores são usados para a construção de uma hierarquia AHP desenvolvida para a identificação e monitoramento de eventos fora dos padrões esperados. O modelo é operacionalizado em uma empresa do setor automotivo, cuja unidade no Brasil está localizada na região metropolitana de Porto Alegre (Brasil).

Valois e Almeida (2009) apresentam um modelo decisório, baseado em técnicas multicritério, que trata do dilema entre terceirizar ou executar com recursos próprios as atividades produtivas dentro de uma organização. Neste estudo, são considerados vários aspectos, como qualidade, custos, credibilidade, aspectos legais, entre outros, que, de alguma forma, impõem restrições à execução de atividades por terceiros. O modelo proposto está fundamentado na técnica SMART. O modelo foi aplicado em uma empresa que atua no ramo da construção civil.

Bandeira, Becker e Rocha (2010) apresentam um modelo multicritério, baseado no AHP para a decisão em grupo, no contexto da priorização de confirmações de reservas de embarque de cargas de exportação, em situação de escassez com falta de contêineres vazios ou espaços nos navios disponíveis.

Também trabalhando com o AHP, porém de forma integrada ao método Delphi de busca de consenso, De Carli, Delamaro e Salomon (2010) propõem e aplicam um modelo para a priorização dos fatores críticos de sucesso na implantação de um projeto de fábrica digital em uma empresa brasileira, os quais foram selecionados por meio de uma pesquisa exploratória a respeito de implantações de sistemas ERP e Product Lifecycle Management.

Ensslin et al. (2010) constroem um modelo para avaliar o desempenho de empresas terceirizadas na área de telecomunicações. Os autores declaram que usam uma abordagem construtivista para a obtenção de informações a partir de entrevistas semiestruturadas com o Gestor de Projetos responsável pela seleção de empresas terceirizadas do setor. Esta abordagem permitiu identificar, organizar, mensurar e integrar os critérios de avaliação. O modelo para tratamento dos dados coletados apresenta as características de agregação da função de utilidade usuais em métodos classificados como MAUT, permitindo ao gerente visualizar o perfil das empresas terceirizadas nos critérios por ele identificados como relevantes para o sucesso de sua organização.

Cristofari Júnior, Paula e Fogliatto (2010) utilizam uma matriz MAUT para a priorização de melhores práticas para a melhoria da gestão do processo de desenvolvimento do produto em uma empresa de desenvolvimento de software.

\subsubsection{Gestão da cadeia de suprimentos}

No conjunto de artigos analisados, foram encontrados seis artigos que lidam com a problemática da gestão da cadeia de suprimentos sob a ótica de uma modelagem multicritério. Quatro destes artigos baseiam-se no método AHP, um deles utiliza o método ELECTRE TRI e outro um modelo multicritério aditivo, sendo, por isso, categorizado como MAUT.

Costa, Soares e Oliveira (2004) apresentam uma metodologia fundamentada no Método Electre Tri para a classificação e escolha de prestadores de serviço para transporte de materiais perigosos. A metodologia proposta neste estudo contém características que permitem considerar a subjetividade inerente ao processo de avaliação de desempenho dos prestadores deste tipo de serviço logístico. O modelo foi operacionalizado por meio da aplicação em um caso em uma empresa distribuidora de combustíveis com rede de postos em todo o Brasil.

Santos Da Rosa, Sellitto e Mendes (2006) expõem um estudo de caso, no qual se usou o AHP para a avaliação do desempenho de dez fornecedores críticos em um sistema de fabricação sob encomenda. Este trabalho contou com a participação de um grupo de especialistas responsáveis pelas aquisições da empresa em estudo, para identificar os critérios de desempenho de fornecedores e julgar a importância relativa dos critérios. Depois desta etapa, foi efetuada uma simulação para a avaliação de dez fornecedores por parte de cinco compradores.

Sellitto e Mendes (2006) integraram o método multicritério AHP ao modelo SCOR de gestão de cadeias de suprimentos, para modelar um processo de avaliação do desempenho de três cadeias de suprimentos situadas no Brasil. Os produtos principais das três cadeias são: materiais de segurança; motores elétricos; e moldes e matrizes para peças injetadas. $\mathrm{O}$ sistema de avaliação foi estruturado com 25 indicadores categóricos avaliados pelos pesquisadores e por gestores das organizações integrantes das três cadeias. $\mathrm{O}$ interesse principal da pesquisa foi propor uma metodologia para a construção de uma estrutura de mensurações capaz de oferecer uma avaliação numérica compreensiva do desempenho de cadeias 
de suprimentos. Este tipo de avaliação, segundo os autores, pode realimentar ações de controle que visem atingir objetivos estratégicos da cadeia de suprimentos e pode ser útil em processos de benchmarking.

Salomon, Marins e Duduch (2009) utilizam o método AHP para a modelagem do processo de seleção de fornecedores em situações nas quais se têm múltiplas decisões. O modelo é aplicado a um caso de seleção de fornecedores de novos equipamentos para duas linhas de montagem de uma fábrica de autopeças localizada no Estado de São Paulo. O modelo considerou o mesmo conjunto de critérios para as duas linhas e permitiu incorporar aspectos da modelagem multidecisor ao problema que se apresentava, inicialmente, como dois modelos independentes.

Zamcopé et al. (2010) desenvolvem um modelo multicritério que tem as características da utilidade aditiva, para a avaliação de operadores logísticos, como instrumento de apoio à elaboração de estratégias de aperfeiçoamento para a cadeia logística de uma pequena empresa paranaense do setor têxtil.

Hofmann (2011) investiga a aplicação do AHP a um exemplo ilustrativo de seleção de fornecedores em um contexto do fenômeno counter trade, no âmbito da gestão da cadeia de suprimentos.

\subsubsection{Gestão da manutenção}

Foram identificados cinco artigos com aplicações da análise multicritério neste tema. O Promethee II foi o método mais utilizado. Os métodos MAUT, Electre I e AHP tiveram uma ocorrência cada um.

Almeida (2005) aborda o problema de seleção de intervalos, ou periodicidade de manutenção preventiva, em um sistema de produção de serviços. Sua análise foi feita por meio de um modelo de decisão multicritério, auxiliado pelo MAUT. A modelagem proposta é aplicada em uma empresa de energia elétrica.

Cavalcante e Almeida (2005) apresentam um modelo de decisão multicritério baseado no método PROMETHEE II que atua em situações de incerteza (não se tem dados de falhas ou estes são pouco confiáveis) e permite estabelecer a periodicidade da manutenção preventiva no sistema de produção. Neste modelo, são levados em conta os parâmetros de custos e confiabilidade. A importância deste trabalho recai no fato de que minimiza ou não deixa que a ausência de dados afete negativamente a estipulação dos tempos de manutenção.

Cavalcante e Seixas Costa (2006) abordam o problema da manutenção preventiva como forma de gerar vantagens competitivas. Estes autores propõem um modelo de apoio multicritério à decisão que usa a metodologia da família PROMETHEE para ajudar ao tomador da decisão na escolha de períodos de tempos ótimos para fazer a manutenção preventiva no processo produtivo. Este trabalho melhora um modelo multicritério existente que trata a gestão da manutenção e faz um tratamento mais cuidadoso nos casos em que o tempo de reparo não pode ser ignorado.

Helmann e Marçal (2007) se apoiam no método multicritério ELECTRE I para auxiliar a Gestão da Manutenção. O objetivo principal deste estudo é a determinação dos equipamentos críticos ao processo produtivo com o propósito de estabelecer políticas de manutenção diferenciadas, a fim de evitar danos humanos, financeiros e ambientais à empresa, aos colaboradores e à sociedade. Para exemplificar o uso do modelo proposto, os autores modelaram uma situação na qual se deve selecionar equipamentos críticos entre seis alternativas quaisquer de um processo de fabricação de embalagens de polpa moldada. A decisão de identificação ocorre a partir dos seguintes critérios de avaliação: riscos de segurança ao ser humano e às instalações; riscos ao meio ambiente; e perdas de produção. Foram realizadas comparações entre pares de equipamentos e a construção das relações de sobreclassificação a partir de índices de concordância e discordância.

Fernandes e Godinho Filho (2010) propõem um modelo em que é usado o método AHP na identificação e seleção de técnicas de manutenção compatíveis ao processo de armazenagem de grãos, mais especificamente de grãos de soja. Neste trabalho, foram consideradas como alternativas de técnicas de seleção: manutenção corretiva; manutenção preventiva; manutenção baseada em condição; e manutenção preditiva. Estas opções de técnicas foram avaliadas de acordo com seu desempenho à luz de seis critérios (segurança, produtividade, qualidade, frequência do problema, taxa de ocupação e manutenção). Os resultados foram processados pelo método AHP, indicando a solução a ser adotada.

\subsubsection{Gestão de projetos}

No âmbito da gestão de projetos, foram identificados cinco artigos, dois baseados nos métodos ELECTRE, um no TODIM e outros dois no AHP.

Mota e Almeida (2007) apresentam o método ELECTRE IV-H, baseado no método ELECTRE IV, desenvolvido para apoiar o processo de priorização de atividades em projetos, nos quais não se pode considerar os pesos dos critérios e, também, para casos em que há apenas critérios suscetíveis de avaliação em escalas ordinais. O método é aplicado a um caso específico de priorização de atividades no âmbito da construção de subestações elétricas.

Alencar, Almeida e Mota (2007) apresentam uma sistemática integrada para a seleção de fornecedores de serviços de gerenciamento de projetos. Esta sistemática incorpora múltiplos critérios de avaliação, com foco nos diferentes objetivos existentes ao longo 
das diferentes etapas do gerenciamento de projetos. Este trabalho baseia-se na modelagem pelos métodos ELECTRE.

Monteiro Gomes e Maranhão (2008) se apoia no método de análise multicritério TODIM para sustentar um processo decisório, relacionado à análise e seleção da melhor opção para destinação das reservas de gás natural, descobertas recentemente no campo de Mexilhão da Bacia de Santos. O trabalho aplicou a técnica de Minimização Heurística da Interdependência entre Critérios (MHIC) sobre um conjunto inicial de 12 critérios, resultando em um conjunto final de 8 critérios.

Padovani, Carvalho e Muscat (2010) aplicam o AHP para a seleção de alternativas para comporem o portfólio de projetos de uma empresa do setor químico. Os autores verificaram que o uso do modelo contribui para o alinhamento estratégico e melhora da troca de informações entre os tomadores de decisão da empresa, além de permitir a alocação de recursos baseada em prioridades.

Dias, Fernstenseifer e Sellitto (2011) apresentam um modelo multicritério baseado no AHP, para a identificação das prioridades estabelecidas por seis supermercados compradores de arroz aos critérios de competição observados no contexto do comércio deste produto.

\subsubsection{Logística de distribuição}

Na pesquisa, foi identificado apenas um artigo que integra a abordagem multicritério e problemas de logística de distribuição. Iañez e Cunha (2006) tratam o problema da tomada de decisão, vinculada à terceirização das atividades logísticas, por meio da seleção de um provedor de serviços logísticos. A modelagem proposta, baseada no método AHP, contemplou três aspectos principais: a análise e a compreensão das oportunidades e riscos relacionados à terceirização das atividades logísticas; a proposição de atributos importantes que podem ser considerados no processo decisório; e o auxílio às empresas na avaliação das diferentes alternativas de provedores de serviços logísticos. A metodologia foi validada por meio de um estudo de caso que envolveu a avaliação de um processo de terceirização das atividades logísticas para uma grande empresa do setor de eletroeletrônicos que possuía, na época da pesquisa, duas unidades industriais no Brasil: em Manaus e no Vale do Paraíba.

\subsubsection{Modelos de estoques}

$\mathrm{Na}$ literatura analisada, foram identificados três estudos, dois utilizaram o método ELECTRE TRI e um foi baseado no AHP.
Herrera e Costa (2005) propõem o uso do ELECTRE TRI para a adaptação da classificação de estoques do Sistema ABC-pareto. A proposta é utilizada para a classificação de itens adquiridos por uma fundação pública de direito privado, localizada no interior do Estado do Rio de Janeiro. O trabalho oferece uma análise de sensibilidade do modelo quanto a inconsistências e incomparabilidades na classificação obtida.

Szajubok, Mota e Almeida (2006) também aplicam o ELECTRE TRI para a classificação de itens em estoque. A modelagem desenvolvida, neste trabalho, é aplicada na classificação de materiais e estoques na indústria da construção civil. O modelo foi aplicado em uma empresa construtora de médio porte do Recife. Este mesmo trabalho usa o método ELECTRE TRI em uma aplicação ao conceito do MRP de forma a apoiar o planejamento e gerenciamento de materiais, conforme mencionado na próxima subseção.

Sellitto, Borchardt e Pereira (2009) apresentam um estudo de caso duplo, no qual foi elaborada uma modelagem baseada no método AHP para a classificação de itens de fornecimento como suporte da definição das estratégias de gestão e colaboração com os fornecedores. O emprego da modelagem resultou em uma classificação diferente daquela que era utilizada pelas organizações investigadas, sendo uma integrante da cadeia automotiva e a outra da cadeia de fabricação de calçados.

\subsubsection{Planejamento das necessidades de materiais}

No contexto do Planejamento das necessidades de materiais, foi identificado e analisado apenas um artigo associado ao uso de métodos multicritério (SZAJUBOK; MOTA; ALMEIDA, 2006), trabalho este já mencionado na subseção anterior referente a modelos de estoque.

\subsubsection{Planejamento de layout}

$\mathrm{Na}$ abordagem do planejamento de layout, auxiliado por técnicas de multicritério, foram encontrados dois trabalhos. Um deles utilizou o ELECTRE e outro usou o AHP. Ambos utilizaram as técnicas como metodologia base para a construção de arranjos físicos.

Herrera e Costa (2001) propõem uma abordagem multicritério, baseada no método ELECTRE TRI, para a classificação e obtenção de graus de proximidade (GP) que expressam a intensidade com que se deve priorizar a aproximação de duas áreas ou estações de trabalho em um arranjo físico. No modelo proposto, os critérios e os seus respectivos graus de importância foram determinados por especialistas. Para avaliação do grau de proximidade das alternativas de posicionamento em cada critério, foram adotadas 
escalas de aceitação e rejeição. O modelo foi testado pelos pesquisadores de forma generalizada em um caso de obtenção de GP de cinco áreas diferentes. O trabalho apresenta um caráter exclusivamente didático, e os julgamentos foram arbitrados com a ideia de expor a operacionalidade do método.

Tortorella e Fogliatto (2008) tratam da utilização do espaço físico de uma fábrica, visando o seu uso eficiente e ótimo, objetivando melhorar o fluxo de materiais, produtos e informação dentro das instalações. Este trabalho combina o uso do método AHP ao método para planejamento do macroespaço SLP (Systematic Layout Planning - Planejamento Sistemático de Layout). O modelo proposto foi colocado em prática na seleção de alternativas de layout para uma empresa do ramo automotivo localizada em Porto Alegre, empresa esta que necessitava ampliar seu parque fabril. Nove alternativas de layout para a empresa foram geradas com o uso do método SLP. Posteriormente, estas alternativas foram avaliadas à luz de sete critérios: Fatores humanos; Nível de re-layout; Custo, Relacionamento entre as SPUs; Linearidade de fluxo; Aproveitamento de área; e Flexibilidade para futuras expansões.

\subsection{Estatísticas do conjunto de artigos pesquisados}

Nesta seção, são apresentadas estatísticas que sintetizam a distribuição da produção científica no tema pesquisado por periódicos e ao longo do tempo.

A Tabela 3 apresenta a lista dos periódicos analisada, na qual se encontra pelo menos um artigo no contexto do trabalho. Esta tabela também informa o período em que foram pesquisados, o qual está associado ao período disponibilizado pelo periódico dentro do recorte temporal 1999-2011. Outro dado presente na tabela é a frequência de artigos encontrados em cada um dos periódicos. O objetivo da Tabela 3 é auxiliar o pesquisador interessado em estudar a interface $P C P \&$ Multicritério na tarefa de identificação dos periódicos que mais apresentam publicações neste contexto e que, portanto, devem ser monitorados com maior frequência.

É importante ressaltar que outros periódicos foram consultados, mas não contêm no intervalo de pesquisa usado (1999 a 2011) artigos relacionados ao tema, entre eles, Revista de Administração Contemporânea (RAC), Brazilian Administrative Review (BAR) e Revista de Administração de Empresas (RAE), periódicos estes que costumam publicar artigos em temáticas envolvendo Planejamento e Controle da Produção.

A Figura 1 apresenta os dados quantitativos da produção científica selecionada no âmbito da pesquisa, agrupados por ano de publicação. Observa-se um aumento considerado de publicações a partir de

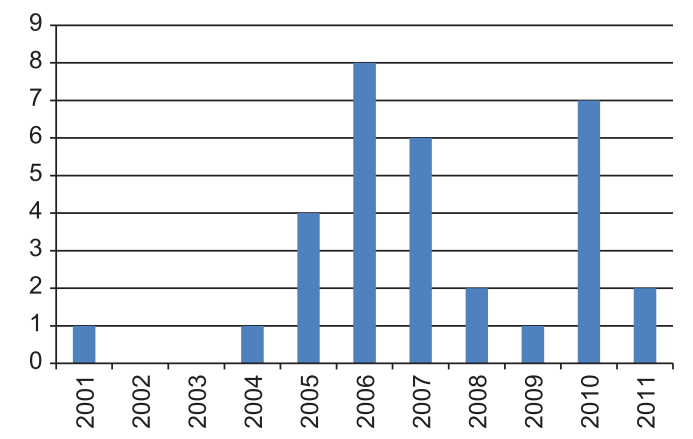

Figura 1. Publicações por ano.

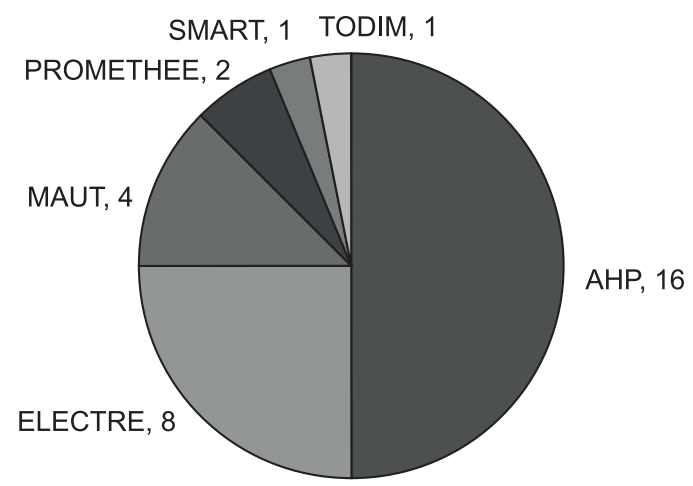

Figura 2. Distribuição dos artigos por método multicritério.

Tabela 3. Periódicos e número de artigos pesquisados.

\begin{tabular}{lllc}
\hline \multicolumn{1}{c}{ Periódico } & \multicolumn{1}{c}{ Sigla } & \multicolumn{1}{c}{ Período } & Número de artigos \\
\hline Produção & Produção & $2002-2011$ & 10 \\
Pesquisa Operacional & PO & $2000-2001$ & 6 \\
Gestão \& Produção & G\&P & $1999-2011$ & 5 \\
Produção On-Line & PO & $2001-2011$ & 3 \\
Brazilian Journal of Operations \& Production Management & BJO\&PM & $2004-2011$ & 2 \\
Gestão Industrial & G.I & $2005-2011$ & 2 \\
Produto \& Produção & P\&P & $2001-2011$ & 1 \\
Pesquisa Operacional para o Desenvolvimento & PODES & $2009-2011$ & 1 \\
Pesquisa \& Desenvolvimento de Engenharia de Produção & P\&D & $2003-2011$ & 1 \\
Revista de Administração Mackenzie & RAM & $2003-2011$ & 1 \\
\hline
\end{tabular}


Tabela 4. Distribuição dos artigos por área do PCP.

\begin{tabular}{|c|c|c|c|}
\hline Temática do PCP & Referências & Método & Setor de aplicação \\
\hline \multirow{9}{*}{$\begin{array}{l}\text { Controle da } \\
\text { Produção }\end{array}$} & Sellitto e Walter (2006) & AHP & Fabricação de máquinas e equipamentos \\
\hline & Salomon e Shimizu (2006) & $\mathrm{AHP}$ & Fabricação de máquinas e equipamentos \\
\hline & Aguiar e Salomon (2007) & AHP & Fabricação de máquinas e equipamentos \\
\hline & Rafaeli e Müller (2007) & AHP & Fabricação de máquinas e equipamentos \\
\hline & Valois e Almeida (2009) & SMART & Construção \\
\hline & Bandeira, Becker e Rocha (2010) & AHP & Transporte aquaviário \\
\hline & $\begin{array}{l}\text { De Carli, Delamaro e } \\
\text { Salomon (2010) }\end{array}$ & AHP & Fabricação de máquinas e equipamentos \\
\hline & Ensslin et al. (2010) & MAUT & Telecomunicações \\
\hline & $\begin{array}{l}\text { Cristofari Júnior, Paula e } \\
\text { Fogliatto (2010) }\end{array}$ & MAUT & $\begin{array}{c}\text { Atividades dos serviços de tecnologia da } \\
\text { informação }\end{array}$ \\
\hline \multirow{6}{*}{$\begin{array}{l}\text { Gestão da cadeia } \\
\text { de suprimentos }\end{array}$} & Costa, Soares e Oliveira (2004) & Electre TRI & Transporte rodoviário \\
\hline & $\begin{array}{c}\text { Santos Da Rosa, Sellitto e } \\
\text { Mendes (2006) }\end{array}$ & AHP & Não especificado \\
\hline & Sellitto e Mendes (2006) & AHP & Fabricação de máquinas e equipamentos \\
\hline & $\begin{array}{l}\text { Salomon, Marins e } \\
\text { Duduch (2009) }\end{array}$ & AHP & Fabricação de máquinas e equipamentos \\
\hline & Zamcopé et al. (2010) & MAUT & Fabricação de produtos têxteis \\
\hline & Hofmann (2011) & AHP & Não especificado \\
\hline \multirow{5}{*}{$\begin{array}{l}\text { Gestão da } \\
\text { Manutenção }\end{array}$} & Almeida (2005) & MAUT & $\begin{array}{c}\text { Produção, transmissão e distribuição de } \\
\text { energia elétrica e equipamentos }\end{array}$ \\
\hline & Cavalcante e Almeida (2005) & Promethee II & $\begin{array}{l}\text { Produção, transmissão e distribuição de } \\
\text { energia elétrica e equipamentos }\end{array}$ \\
\hline & Cavalcante e Seixas Costa (2006) & Promethee II & Transporte aéreo \\
\hline & Helmann e Marçal (2007) & Electre I & $\begin{array}{l}\text { Fabricação de produtos diversos } \\
\text { (embalagens) }\end{array}$ \\
\hline & $\begin{array}{c}\text { Fernandes e } \\
\text { Godinho Filho (2010) }\end{array}$ & AHP & $\begin{array}{l}\text { Agricultura, pecuária e serviços } \\
\text { relacionados }\end{array}$ \\
\hline \multirow{5}{*}{$\begin{array}{l}\text { Gestão de } \\
\text { Projetos }\end{array}$} & Mota e Almeida (2007) & Electre IV & Construção \\
\hline & Alencar, Almeida e Mota (2007) & Electre & Construção \\
\hline & $\begin{array}{l}\text { Monteiro Gomes e } \\
\text { Maranhão (2008) }\end{array}$ & TODIM & Extração de petróleo e gás natural \\
\hline & $\begin{array}{l}\text { Padovani, Carvalho e } \\
\text { Muscat (2010) }\end{array}$ & AHP & Fabricação de produtos químicos \\
\hline & $\begin{array}{l}\text { Dias, Fernstenseifer e } \\
\text { Sellitto (2011) }\end{array}$ & AHP & Comércio varejista (supermercado) \\
\hline $\begin{array}{l}\text { Logística de } \\
\text { distribuição }\end{array}$ & Iañez e Cunha (2006) & AHP & $\begin{array}{c}\text { Fabricação de máquinas, aparelhos e } \\
\text { materiais elétricos }\end{array}$ \\
\hline \multirow{3}{*}{$\begin{array}{l}\text { Modelos de } \\
\text { estoques }\end{array}$} & Herrera e Costa (2005) & Electre Tri & $\begin{array}{c}\text { Administração pública, defesa e } \\
\text { seguridade social }\end{array}$ \\
\hline & Szajubok, Mota e Almeida (2006) & Electre Tri & Construção \\
\hline & $\begin{array}{l}\text { Sellitto, Borchardt e } \\
\text { Pereira (2009) }\end{array}$ & AHP & $\begin{array}{c}\text { Fabricação de veículos automotores, } \\
\text { reboques e carrocerias / Preparação de } \\
\text { couros e fabricação de artefatos de couro, } \\
\text { artigos para viagem e calçados }\end{array}$ \\
\hline $\begin{array}{c}\text { Planejamento das } \\
\text { necessidades de } \\
\text { materiais }\end{array}$ & Szajubok, Mota e Almeida (2006) & Electre Tri & Construção \\
\hline \multirow{2}{*}{$\begin{array}{l}\text { Planejamento de } \\
\text { Layout }\end{array}$} & Herrera e Costa (2001) & Electre Tri & Não especificado \\
\hline & Tortorella e Fogliatto (2008) & AHP & $\begin{array}{l}\text { Fabricação de veículos automotores, } \\
\text { reboques e carrocerias }\end{array}$ \\
\hline
\end{tabular}


2005, com picos em 2006 e em 2010 (sete artigos em cada ano).

\subsection{Síntese dos resultados}

A Tabela 4 foi estruturada para apresentar uma visão sistêmica e panorâmica dos estudos analisados. Os dados desta tabela permitem identificar a distribuição de artigos por tema pesquisado, assim, com a distribuição dos métodos multicritério aplicados por área do PCP. Os dados referentes à última coluna (setor de aplicação) foram elaborados com base na tabela "Classificação Nacional de Atividades Econômicas/CNAE", obtida no sítio da Receita Federal (http://www.receita.fazenda.gov.br/pessoajuridica/ cnaefiscal/cnaef.htm). Esta classificação foi adotada por ser aquela adotada pelo Conselho Nacional de Desenvolvimento Científico e Tecnológico (CNPq) para o cadastro da produção científica e intelectual no sistema de Currículo Lattes.

Contrastando as informações oferecidas na Tabela 4 com as informações presentes na Tabela 1, é possível concluir que há várias áreas de PCP para as quais ainda não se aplicou, na base de periódicos e no horizonte de tempo pesquisados, alguma modelagem multicritério: Job shop; localização; planejamento agregado; planejamento da capacidade; previsão de demanda; programa mestre de produção (MPS); e programação da produção. Esta tabela também permite obter as áreas de PCP mais exploradas no contexto da pesquisa, que são controle da produção, gestão da cadeia de suprimentos, gestão de projetos e gestão da manutenção. Com respeito às atividades produtivas nas quais foram identificadas aplicações multicritério no âmbito da presente pesquisa, destaca-se a "Fabricação de máquinas e equipamentos" com sete artigos. Outro setor com destaque foi o de construção, com cinco artigos. De uma forma geral, predominaram as aplicações associadas à produção de bens de capital, totalizando 13 artigos.

A Figura 2 foi elaborada com base na Tabela 4 e sintetiza a quantidade de artigos por método multicritério. Analisando as informações desta figura, conclui-se que uma quantidade significativa (75\%) das modelagens efetuadas no contexto de PCP e multicritério adotam os métodos AHP (18 artigos) e ELECTRE (oito artigos). Somente o método AHP foi aplicado em 50\% do total de artigos.

\section{Conclusão}

Este trabalho apresenta uma pesquisa sistemática sobre a produção científica publicada em periódicos brasileiros no âmbito da aplicação Auxílio Multicritério à Decisão à modelagem de problemas de PCP. Trinta e dois artigos, de 10 periódicos científicos publicados no Brasil, foram selecionados e analisados criteriosamente. A pesquisa cobriu os periódicos registrados no Scielo, além de alguns periódicos publicados no Brasil não indexados no Scielo, mas relacionados a associações científicas de prestígio, que cobrem a temática do PCP.

Dentre os fatos e tendências observadas, destaca-se que:

- O método AHP é o que apresenta maior frequência na modelagem dos problemas, visto que 16 dos 32 dos artigos pesquisados adotam este método;

• "Controle da produção", "Gestão da Cadeia de Suprimentos" e "Gestão da Manutenção" são os temas de PCP com maior frequência de modelagens multicritério nos artigos analisados;

- Tópicos como "Job shop", "Previsão de demanda", "Planejamento agregado", "Plano mestre de produção (MPS)", "Planejamento da capacidade" e localização ainda não foram abordados sob a ótica multicritério; e

- As aplicações de métodos MCDA predominantes estão associadas à produção de bens de capital totalizando, principalmente, "Fabricação de máquinas e equipamentos" e "construção".

Apesar desta pesquisa não ter a intenção de ser exaustiva, acredita-se que os artigos selecionados compreendem uma representativa e significante base sobre a pesquisa científica que vem sendo realizada por pesquisadores, e que a análise desta fornece uma base confiável do estudo da arte realizado na interface do PCP com o MCDA. Como é impossível ler tudo o que existe e que está disponível sobre algum assunto, a presente pesquisa tem suas limitações. $\mathrm{O}$ uso de uma base eletrônica de dados oculta artigos de outras bases que podem ser relevantes para a revisão do tópico, assim como o uso das expressões booleanas adotadas para o processo de seleção não podem garantir que outros artigos, na mesma base, lidando com o tema, existam, só que com outras expressões/termos. A consideração de apenas artigos publicados em periódicos científicos também é uma limitação, dado que conceitos importantes podem estar divulgados na "Gray literature", assim como o intervalo de tempo de 13 anos. Neste sentido, pesquisas futuras são sugeridas a partir da remoção de algumas destas limitações, por exemplo, com a inclusão de teses de doutorado e dissertações de mestrado defendidas no Brasil.

Também se sugere como temas para trabalhos futuros a replicação desta pesquisa em contexto internacional, com a comparação dos resultados aos aqui apresentados, que exprimem uma realidade particular associada à brasileira. A incorporação dos métodos MCDM e o desenvolvimento de modelos multicritério em contextos ainda não veiculados na literatura brasileira (e.g., previsão de demanda, 
planejamento da capacidade, planejamento agregado, job shop e programa mestre de produção) também são sugeridos.

\section{Referências}

AGUIAR, D. C.; SALOMON, V. A. P. Avaliação da prevenção de falhas em processos utilizando métodos de tomada de decisão. Produção, v. 17, n. 3, p. 502-519, 2007. http://dx.doi.org/10.1590/S0103-65132007000300008

ALENCAR, L. H.; ALMEIDA, A. T.; MOTA, C. M. M. Sistemática proposta para seleção de fornecedores em gestão de projetos. Gestão \& Produção, v. 14, n. 3, p. 477-487, 2007. http://dx.doi.org/10.1590/ S0104-530X2007000300005

ALMEIDA, A. T. Modelagem Multicritério para Seleção de Intervalos de Manutenção Preventiva Baseada na Teoria da Utilidade Multiatributo. Pesquisa Operacional, v. 25, n. 1, p. 69-81, 2005. http://dx.doi.org/10.1590/ S0101-74382005000100005

BANA E COSTA, C. A.; VANSNICK, J. C. MACBETH - An interactive path towards the construction of cardinal value functions. International Transactions in Operational Research, v. 1, n. 4, p. 489-500, 1994. http://dx.doi. org/10.1016/0969-6016(94)90010-8

BANDEIRA, D. L.; BECKER, J. L.; ROCHA, A. K. Sistemática multicritério para priorização de embarques marítimos. RAM. Revista de Administração Mackenzie, v. 11, p. 107-130, 2010. http://dx.doi. org/10.1590/S1678-69712010000600007

BEHZADIAN, M. et al. Promethee: A comprehensive literature review on methodologies and applications. European Journal of Operational Research, v. 200, n. 1, p. 198-215, 2010. http://dx.doi.org/10.1016/j. ejor.2009.01.021

BRANS, J. P.; MARESCHAL, B.; VINCKE, P. Promethee: a New Family of Outranking Methods in Multicriteria Analysis. Washington: North-Holland, 1984. p. 477-490.

BRANS, J. P.; VINCKE, P.; MARESCHAL, B. How to select and how to rank projects: The Promethee method. European Journal of Operational Research, v. 24, n. 2, p. 228-238, 1986. http://dx.doi. org/10.1016/0377-2217(86)90044-5

BUFFA, E. S. Production-inventory systems: planning and control. New York, 1976.

CAVAlCANTE, C. A. V.; ALMEIDA, A. T. Modelo Multicritério de Apoio a Decisão para o Planejamento de Manutenção Preventiva Utilizando PROMETHEE II em Situações de Incerteza. Pesquisa Operacional, v. 25, n. 2, p. 279-296, 2005. http://dx.doi.org/10.1590/ S0101-74382005000200007

CAVAlCANTE, C. A. V.; SEIXAS COSTA, A. P. C. Multicriteria Model of Preventive Maintenance. Brazilian Journal of Operations \& Production Management, v. 3, n. 1, p. 71-86, 2006.

CHAPMAN, S. N. Fundamentals of production planning and control. New York: Prentice Hall, 2006.

CLÍMACO, J. C. N. et al. Supporting collaborative multicriteria evaluation: the VIP Analysis plug-In for decision deck. International Journal of Decision Support
System Technology, v. 1, n. 4, p. 1-15, 2009. http:// dx.doi.org/10.4018/jdsst.2009062601

CORREA, H. L.; GIANESE, I. G. N.; CAON, M. Planejamento, programação e controle da produção. São Paulo: Atlas, 2007.

COSTA, H. G. et al. ELECTRE TRI applied to costumers satisfaction evaluation. Produção, v. 17, n. 2, p. 230-245, 2007. http://dx.doi.org/10.1590/ S0103-65132007000200002

COSTA, H. G.; SOARES, A. C.; OLIVEIRA, P. F. Avaliação de transportadoras de materiais perigosos utilizando o método electre tri. Gestão \& Produção, v. 11, n. 4, p. 221-229, 2004.

CRISTOFARI JÚNIOR, C. A.; PAULA, I. C. D.; FOGLIATTO, F. S. Método de análise de maturidade e priorização de melhorias na gestão do Processo de Desenvolvimento de Produtos. Produção, v. 20, p. 359-377, 2010. http://dx.doi.org/10.1590/ S0103-65132010005000039

DE CARLI, P. C.; DELAMARO, M. C.; SALOMON, V. A. P. Identificação e priorização dos fatores críticos de sucesso na implantação de fábrica digital. Produção, v. 20, n. 4, 2010. http://dx.doi.org/10.1590/ S0103-65132010005000037

DIAS, M. F. P.; FERNSTENSEIFER, J. E.; SELLITTO, M. A. Análise multicriterial em estratégia de operações: estudo de caso com compradores de arroz de seis redes supermercadistas. Produção online, v. 11, n. 3 , p. 707-734, 2011.

EDWARDS, W. How to use multiattribute utility measurement for social decisionmaking. IEEE Transactions on Systems, Man and Cybernetics, v. SMC-7, n. 5, p. 326-340, 1977. http://dx.doi. org/10.1109/TSMC.1977.4309720

EHRGOTT, M.; FIGUEIRA, J.; GRECO, S. Trends in multiple criteria decision analysis. Berlin: Springer, 2010. 412 p. (International Series in Operations Research \& Management Science).

ELSAYED, E. A.; BOUCHER, T. O. Analysis and Control of Production Systems. New York: Prentice Hall, 1994. 450 p.

ENSSLIN, L. et al. Avaliação do Desempenho de Empresas Tercerizadas com Uso da Metodologia Multicritério de Apoio à Decisão-Construtivista. Pesquisa Operacional, v. 30, n. 1, p. 125-152, 2010. http://dx.doi.org/10.1590/ S0101-74382010000100007

FERNANDES, F. C. F.; GODINHO FILHO, M. Planejamento e controle da produção: Dos fundamentos ao essencial. São Paulo: Atlas, 2010. 296 p.

FETSCHERIN, M.; VOSS, H.; GUGLER, P. 30 Years of foreign direct investment to China: An interdisciplinary literature review. International Business Review, v. 19, n. 3, p. 235-246, 2010. http://dx.doi.org/10.1016/j. ibusrev.2009.12.002

FIGUEIRA, J.; GRECO, S.; EHRGOTT, M. Multiple criteria decision analysis: state of the art surveys. New York: Springer, 2005. 1004 p. (International Series in Operations Research \& Management Science).

FISHBURN, P. C. Utility theory for decision making New York: Wiley, 1970. xiv, 234 p. (Operations Research Society of America Publications in operations research). 
GOMES, C. F. S. Using MCDA methods THOR in an application for outranking the ballast water management options. Pesquisa Operacional, v. 25, n. 1, p. 11-28, 2005. http://dx.doi.org/10.1590/ S0101-74382005000100002

GOMES, L. F.; LIMA, M. From modelling individual preferences to multicriteria ranking of discrete alternatives: a look at prospect theory and the additive difference model. Foundations of Computing and Decisions Sciences, v. 17, n. 3, p. 171-184, 1992.

HELMANN, K. S.; MARÇAL, R. F. M. Método Multicritério de Apoio à Decisão na Gestão da Manutenção: Aplicação do Método ELECTRE I na Seleção de Equipamentos Críticos para Processo. Gestão Industrial, v. 3, n. 1, p. 123-133, 2007.

HERRERA, W. D. M.; COSTA, H. G. Contribuições da análise multicritério à obtenção de graus de proximidade no projeto de arranjos físicos. Produto \& Produção, v. 5, n. 3, p. 48-60, 2001.

HERRERA, W. D. M.; COSTA, H. G. Uma Forma de Clasificación Multicriterio - ABC. Pesquisa e Desenvolvimento Engenharia de Produção, n. 4, p. 55-66, 2005.

HINLOOPEN, E.; NIJKAMP, P.; RIETVELD, P. The Regime method: a new multicriteria method. Essays and surveys on multiple criteria decision making. Lecture Notes in in Economics and Mathematical Systems, v. 209, p. 146-155, 1983. http://dx.doi. org/10.1007/978-3-642-46473-7_13

HOFMANN, E. Compensation and buy-back deals in supply chains: analyzing strategic decision areas by using AHP. Brazilian Journal of Operations \& Production Management, v. 8, n. 2, p. 17-49, 2011. http://dx.doi. org/10.4322/bjopm.2012.002

HWANG, C. L.; YOON, K. Multiple attribute decision making methods and applications: a state-ofthe-art survey. Berlin: Springer, 1981. http://dx.doi. org/10.1007/978-3-642-48318-9

IAÑEZ, M. M.; CUNHA, C. B. Uma metodologia para a seleção de um provedor de serviços logísticos. Produção, v. 16, n. 3, p. 394-412, 2006. http://dx.doi.org/10.1590/ S0103-65132006000300004

KEENEY, R. L.; RAIFFA, H. Decisions with Multiple Objectives: preferences and value tradeoffs. New York: John Willey \& Sons, 1976. 569 p.

LARICHEV, O.; MOSHKOVICH, H. M. Verbal decision analysis for unstructured problems. Boston: Kluwer Academic Publishers, 1997.

LARICHEV, O. I.; MOSHKOVICH, H. M. ZAPROS-LM - a method and system for rank-ordering of multiattribute alternatives. European Journal of Operations Research, v. 82, n. 1, p. 503-552, 1995. http://dx.doi. org/10.1016/0377-2217(93)E0143-L

LUSTOZA, L. et al. Planejamento e Controle da Produção. Rio de Janeiro: Campus-Elsevier, 2008.

MARICHALA, J.-L.; MEYERB, P.; ROUBENS, M. Sorting multi-attribute alternatives: The TOMASO method. Computers \& Operations Research, v. 32, n. 4, p. 861-877, 2006. http://dx.doi.org/10.1016/j. cor.2003.09.002
MOTA, C. M. M.; ALMEIDA, A. T. Método multicritério ELECTRE IV-H para priorização de atividades em projetos. Pesquisa Operacional, v. 27, n. 2, p. 247-269, 2007. http://dx.doi.org/10.1590/S0101-74382007000200004

MONTEIRO GOMES, L. F. A.; MARANHÃO, F. J. C. A Exploração de Gás Natural em Mexilhão: Análise Multicritério pelo Método TODIM. Pesquisa Operacional, v. 28, n. 3, p. 491-509, 2008.

MOUSSEAU, V.; SLOWINSKI, R.; ZIELNIEWICZ, P. A user-oriented implementation of the ELECTRE-TRI method integrating preference elicitation support. Computers \& Operations Research, v. 27, n. 7, p. 757-777, 2000. http://dx.doi.org/10.1016/ S0305-0548(99)00117-3

NGAI, E. W. T.; WAT, F. K. T. A literature review and classification of electronic commerce research. Information \& Management, v. 29, p. 415-429, 2002. http://dx.doi.org/10.1016/S0378-7206(01)00107-0

NGAI, E. W. T.; XIU, L.; CHAU, D. C. K. Application of data mining techniques in customer relationship management: A literature review and classification. Expert Systems with Applications, v. 36, p. 2592-2602, 2009. http:// dx.doi.org/10.1016/j.eswa.2008.02.021

NORD, J. H.; NORD, G. D. MIS Research: Journal status assessment and analysis.Information \& Management, v. 29, n. 29-42, 1995.

PADOVANI, M.; CARVALHO, M. M. D.; MUSCAT, A. R. N. Seleção e alocação de recursos em portfólio de projetos: estudo de caso no setor químico. Gestão \& Produção, v. 17, p. 157-180, 2010. http://dx.doi. org/10.1590/S0104-530X2010000100013

POLATIDIS, H. et al. Selecting an appropriate multi-criteria decision analysis technique for renewable energy planning. Energy Sources, Part B, v. 1, p. 181-193, 2006.

RAFAELI, L.; MÜLLER, C. J. Estruturação de um índice consolidado de desempenho utilizando o AHP. Gestão \& Produção, v. 14, n. 2, p. 363-377, 2007. http://dx.doi. org/10.1590/S0104-530X2007000200013

ROTHSTEIN, H. R.; HOPEWELL, S. Grey Literature. In: COOPER, H. et al. (Eds.). The Handbook of Research Synthesis and Meta-Analysis. New York: Russel Sage Foundation, 2009. p. 103-125. Grey Literature.

ROWLEY, J.; SLACK, F. Conducting a literature review. Management Research News, v. 27, n. 6, p. 31-39, 2004. http://dx.doi.org/10.1108/01409170410784185

ROY, B. Classement et choix en presence de points de vue multiples (la methode ELECTRE). Lausanne Presses Polytechiniques et Universitaires Romandes, 1968.

ROY, B. Decision-aid and decision-making. European Journal of Operational Research, v. 45, n. 2-3, p. 324-331, 1990. http://dx.doi. org/10.1016/0377-2217(90)90196-I

ROY, B.; BERTIER, P. M. La methode ELECTRE II: Une methode de classement en presence de criteres multiples. Paris: SEMA (Metra International) Paris, 1971. p. 45. PMid:5161467.

ROY, B.; HUGONNARD, J. C. Classement des prolongements de lignes de stations en banlieu parisienne. Cahiers du LAMSADE. Paris: Université Dauphine et RATP, 1981. 
ROY, B. M.; SKALKA, J. ELECTRE IS: Aspécts methodologiques et guide d'utilization. Cahier du LAMSADE. Paris: Université de Paris-Dauphine, 1985

RUSSOMANO, V. H. PCP - Planejamento e controle da produção. Rio de Janeiro: Pioneira, 1996. 320 p. PMid:8850128.

SAATY, T. L. A scaling method for priorities in hierarchical structures. Journal of Mathematical Psychology, v. 15, n. 3, p. 234-281, 1977. http://dx.doi. org/10.1016/0022-2496(77)90033-5

SAATY, T. L. The Analytic Hierarquic Process. Pittsburg: RWS Publications, 1980.

SAATY, T. L. Decision Making with Dependence and Feedback: The Analytic Network Process. Pittsburg: RWS, 1996.

SALOMON, V. A. P.; MARINS, F.; DUDUCH, M. Tomada de Decisões Múltiplas Aplicada à Seleção de Fornecedores de Equipamentos de uma Linha de Montagem em uma Fábrica de Autopeças. Pesquisa Operacional para o Desenvolvimento, v. 1, n. 3, p. 208-217, 2009.

SALOMON, V. A. P.; SHIMIZU, T. Utilização de Matrizes de Julgamentos na Análise do Controle da Produção. Gestão Industrial, v. 2, n. 1, p. 69-77, 2006.

SANTOS DA ROSA, E. P.; SELLITTO, M. A.; MENDES, L. W. Avaliação multicriterial de desempenho e separação em aglomerados de fornecedores críticos de uma manufatura OKP. Produção, v. 16, n. 3, p. 413-428, 2006.

SELLITTO, M. A.; BORCHARDT, M.; PEREIRA, G. M. Classificação estratégica de fornecimentos de duas operações de manufatura: uma na cadeia automotiva e outra na indústria calçadista. Gestão Industrial, v. 5, n. 2, p. 117-133, 2009.

SELLITTO, M. A.; MENDES, L. W. Avaliação comparativa do desempenho de três cadeias de suprimentos em manufatura. Produção, v. 16, n. 3, p. 552-568, 2006. http://dx.doi.org/10.1590/S0103-65132006000300015

SELLITTO, M. A.; WALTER, C. Avaliação do desempenho de uma manufatura de equipamentos eletrônicos segundo critérios de competição. Produção, v. 16, n. 1, p. 34-47, 2006. http://dx.doi.org/10.1590/ S0103-65132006000100004

SIPPER, D.; BULFIN, R. Production: Planning, Control and Integration. New York: McGraw-Hill, 1997.
SUBRAMANIAN, N.; RAMANATHAN, R. A review of applications of Analytic Hierarchy Process in operations management. International Journal of Production Economics, vol. 138, n. 2, p. 215-241, 2012. http:// dx.doi.org/10.1016/j.ijpe.2012.03.036

SZAJUBOK, N. K.; MOTA, C. M. M.; ALMEIDA, A. T. Uso do Método Multicritério ELECTRE TRI para Classificação de Estoques na Construção Civil. Pesquisa Operacional, v. 26, n. 3, p. 625-648, 2006. http://dx.doi. org/10.1590/S0101-74382006000300010

TAVARES NETO, R. F.; GODINHO FILHO, M. Literature review regarding Ant Colony Optimization applied to scheduling problems: Guidelines forfor implementation and directions for future research. Engineering Applications of Artificial Intelligence, v. 26, n. 1, p. 150-161, 2012. http://dx.doi.org/10.1016/j. engappai.2012.03.011

TORTORELLA, G. L.; FOGLIATTO, F. S. Planejamento sistemático de layout com apoio de análise de decisão multicritério. Produção, v. 18, n. 3, p. 609-624, 2008. http://dx.doi.org/10.1590/S0103-65132008000300015

TUBINO, D. R. Planejamento e controle da produção: teoria e prática. São Paulo: Atlas, 2009.

VALOIS, Ú.; ALMEIDA, A. T. Modelo de apoio à decisão multicritério para terceirização de atividades produtivas baseado no método SMARTS. Produção, v. 19, n. 2, p. 249-260, 2009. http://dx.doi.org/10.1590/ S0103-65132009000200003

VAN RAAN, A. F. J. The use of bibliometric analysis in research performance assessment and monitoring of interdisciplinary scientific developments. Technikfolgenabschätzung. Theorie und Praxis, v. 12, n. 1, p. 20-29, 2003.

VOLLMANN, T. et al. Manufacturing planning and control systems for supply chain management : the definitive guide for professionals. New York: McGrawHill, 2004. 598 p.

YU, W. ELECTRE TRI - Aspects Methodologiques et Guide d'Utilisation. Document du LAMSADE. Paris: Université de Paris-Dauphine, 1992.

ZAMCOPÉ, F. C. et al. Modelo para avaliar o desempenho de operadores logísticos: um estudo de caso na indústria têxtil. Gestão \& Produção, v. 17, p. 693-705, 2010. http://dx.doi.org/10.1590/S0104-530X2010000400005 